\title{
Development of an Intelligent Intruder Alert System
}

\author{
Oyelakin K. Akinloye ${ }^{1,2^{*}}$ and Egbuwalo Shadrack A. ${ }^{2}$ \\ ${ }^{I}$ Space Research Group, Physics Department, Federal University of Technology, \\ Akure, Ondo State, Nigeria. \\ ${ }^{2}$ Physics Unit, Department of Science Laboratory Technology, Rufus Giwa \\ Polytechnic, Owo, Ondo State, Nigeria. \\ *oyelakin13@yahoo.com
}

\begin{abstract}
Threat of burglary and high level of insecurity of lives and properties in the society were found to be sources of concerns to the general populace. Hence, the Development of an Electronic Intelligent Intruder Alert System that deters or frightens a potential burglar or intruder and alert residents. The Sensor, Amplifier, Latching and Alerting units were separately designed, constructed using electronic components and tested before and after casing. It was evident - from the results - that this system can sense/detect the opening/entrance through a door or area under surveillance and sound Alert to inform/warn residence of the presence of an intruder and scares burglars. Hence, the system fulfilled the aim of its production.
\end{abstract}

Keywords: Burglar, Insecurity, Intruder, Intelligent, Alert

\section{Introduction}

Interestingly enough, the concept of burglar alarms dates back thousands of years to a time when dogs were used to warn humans of an intruder like today's burglar. Their barkings used to be the alarms that alerted the people around them that something might be amiss.

The percentage of burglary and crime has increased almost all over the world. Law enforcement agencies have begun to over staff just to deal with the rapid increase in crime. There are many precautions that one can take to safe guard their home or office from such crimes. Keeping a highly trained watch dog is a good option as they are effective in detecting and scaring away potential offenders. These days the most popular item being used to fight and safe guard against this is a burglar alarm or a security system. These come in a large variety of brand names, each one offering features that allow you to keep your home or office secured [1]. Technology has not been left out in the fight against insecurity. Door entry systems is one such hi tech equipments deployed by man to keep him safe. There are very many different types of this system in the market today [2].

An Alert System is one that is able to quickly notice any unusual and potentially dangerous/difficult situation and sounds an alarm as an indication of detection of threat.

Alarms as the name implies are used to alert in the event of any situation that is a threat. There are several kinds of alarms [3]. A security alarm is a system designed to detect intrusion - unauthorized entry - into a building or area. Security alarms are used in residential, commercial, industrial, and military properties for protection against burglary (theft) or property damage, as well as personal protection against intruders. Car alarms likewise protect vehicles and their contents. Prisons also use security systems for control of inmates [4].

Nowadays, closed circuit televisions are incorporated to burglar alarms to detect the presence of unauthorized persons. The output of which is normally a siren or loud speaker alarms to telephone automatic dialers or lighting systems. This serves the function of 
alerting the neighbors of possible intrusion and also serves as a signal to the police [5]. In a related paper titled "Design and Construction of a World-Wide-Mobile-Call-Back Burglar Alarm System" K. A. Oyelakin (2014) described the construction of a burglar alarm system that is capable of calling property owner's mobile phone anywhere (s)he may be in the world and suitable for residential or official property, supermarket, artifacts in museums, jewelry stores and art galleries security [4].

Though, attractive, beautiful and sophisticated as they may be, most of the times, prices of these items are, more often than not, beyond the reach of common people. Hence, the obligation to Design, Construct and Evaluate a cheap Intelligent Intruder Alert System that performs relatively equal to the expensive ones.

\section{Materials and Methods}

Resistors, Capacitors, Light Emitting Diode (LED), Potentiometer, Transistors, Integrated Circuits (IC) among others, were the components used in both the Design and the Construction of this Intelligent Intruder Alert System.

We employed modular approach in this work, thus, in this project considerations were given to the Sensor, Amplifier, Latch and alert sections and the functional block diagram of Figure 1 below shows the interconnection between these blocks.

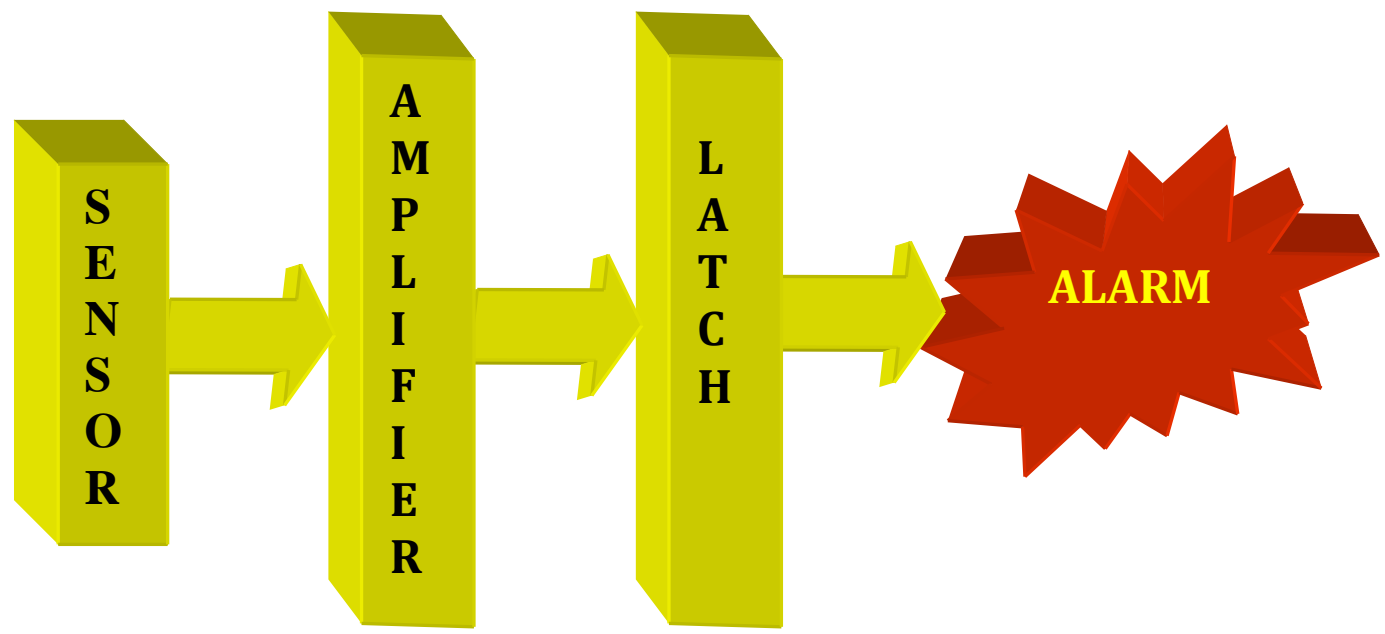

Figure 1. Block Diagram of Intelligent Intruder Alert System

Each section of the circuit that performs a specific function is examined as follow:

\subsection{Sensor}

The sensor unit is divided into two parts, vis:

- Light Emitting section

- Receiver section

\subsubsection{Light Emitting Section}

The Light Emitting section of the sensor unit of this project employed the use of light emitting diode (LED) to produce the required light ray connected as shown in figure 2. 


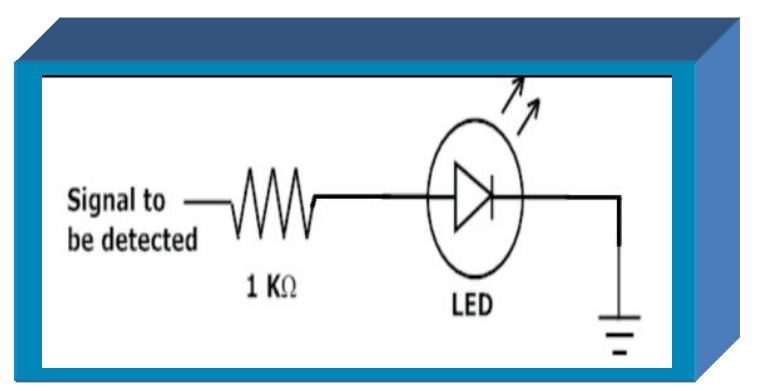

Figure 2. Light Emitting Circuit

\subsubsection{Receiver Section}

We used a combination of Light Dependent Resistor (LDR) - a variable resistor whose resistance value is determined by the level of light ray incident on its surface - and $10 \mathrm{~K}$ potentiometer to construct a voltage divider circuit; as in fig. 3, whose output serves as input to the Operational Amplifier (Op-Amp)

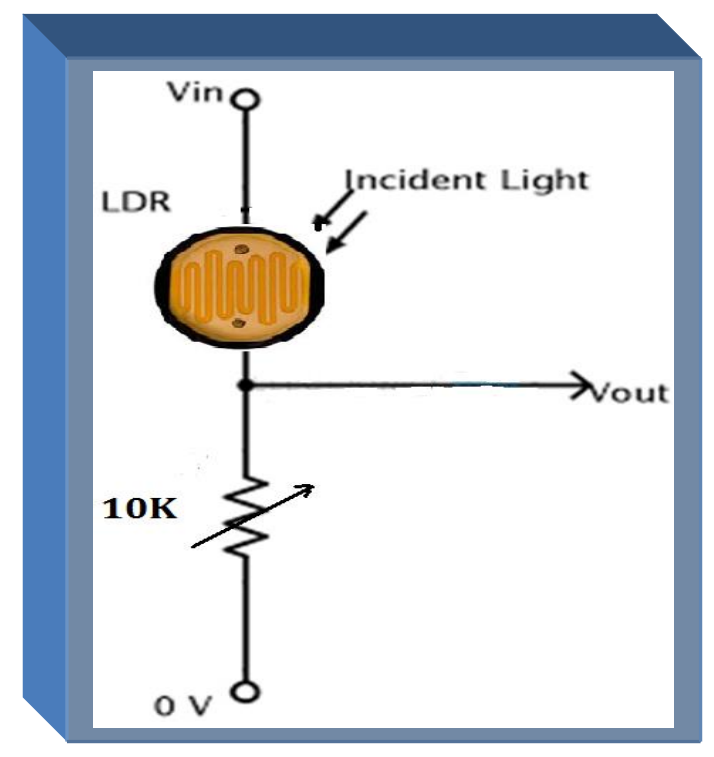

Figure 3. Voltage Divider Circuit

\subsection{Amplifier Section}

The amplifier stage was built around an Op-Amp. The circuit arrangement is such that it can amplify an input signal up to 200 times the original value.

The basic function of the amplifier section; here, is to amplify the input signal from the output of the voltage divider to get the input signals to up to 200 times its original value and pass it on to the latch section of the Intelligent Intruder Alert System for further processing. 


\subsection{Latching Section}

The basic function of latching stage of the circuit, which used a flip-flop Integrated Circuit (IC), is to control the activation or deactivation that is, Setting or Resetting of alert system. Once triggered and a LOW signal is applied at both the RESET and SET inputs, the flip-flop maintains the previous state of the output until the set input goes high. Once an interruption of sensor's light ray occur and the output is amplified and sent to the latch, the latch keeps the alarm continuously ringing until the reset button is pressed. The schematic diagram of the trigger unit:

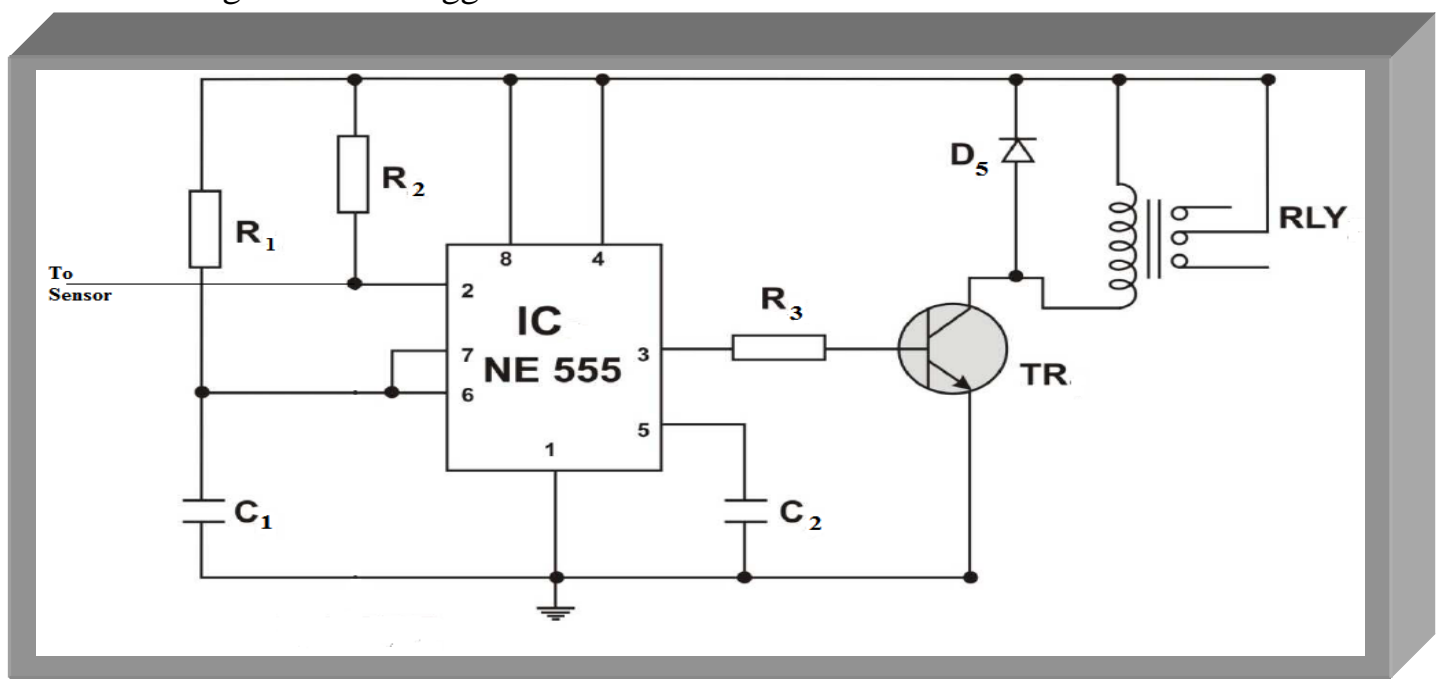

Figure 4. Circuit Diagram of the Trigger Unit

As shown in figure 4 above, the schematic diagram of the trigger unit consist of 3 major components which are NE555 timer (IC), Transistor (TR) and a relay (RLY). The 555 timer (IC) produces a trigger current which comes out through its pin 3 whenever pin 2 is activated through the sensor. Pins 4 and 8 are connected to positive power supply while pin 1 is grounded. $\mathrm{R} 1$ and $\mathrm{C} 1$ determines the time out period of the 555 timer (i.e. the period at which the alarm sound) while R2 determines the sensitivity of the sensor. The output from pin3 (trigger current) is amplified by transistor (TR). R3 act as base resistor to TR which is operating in common emitter mode. The output current from transistor (TR) causes the relay (RLY) to operate thereby switching on the alarm/amplifier unit to power supply for duration of time determined by the time out period of the 555 timer (IC). D5 acts as a flyback or snubber diode protecting the transistor (TR) from Back-EMF generated by the relay coils. The 555 timer in this unit operates in a mono stable mode. 


\subsection{Alarm Section}

An alarm unit was needed to produce audible sound so as to signify the activation of the alert system, that is, presence of an intruder on the property being monitored.

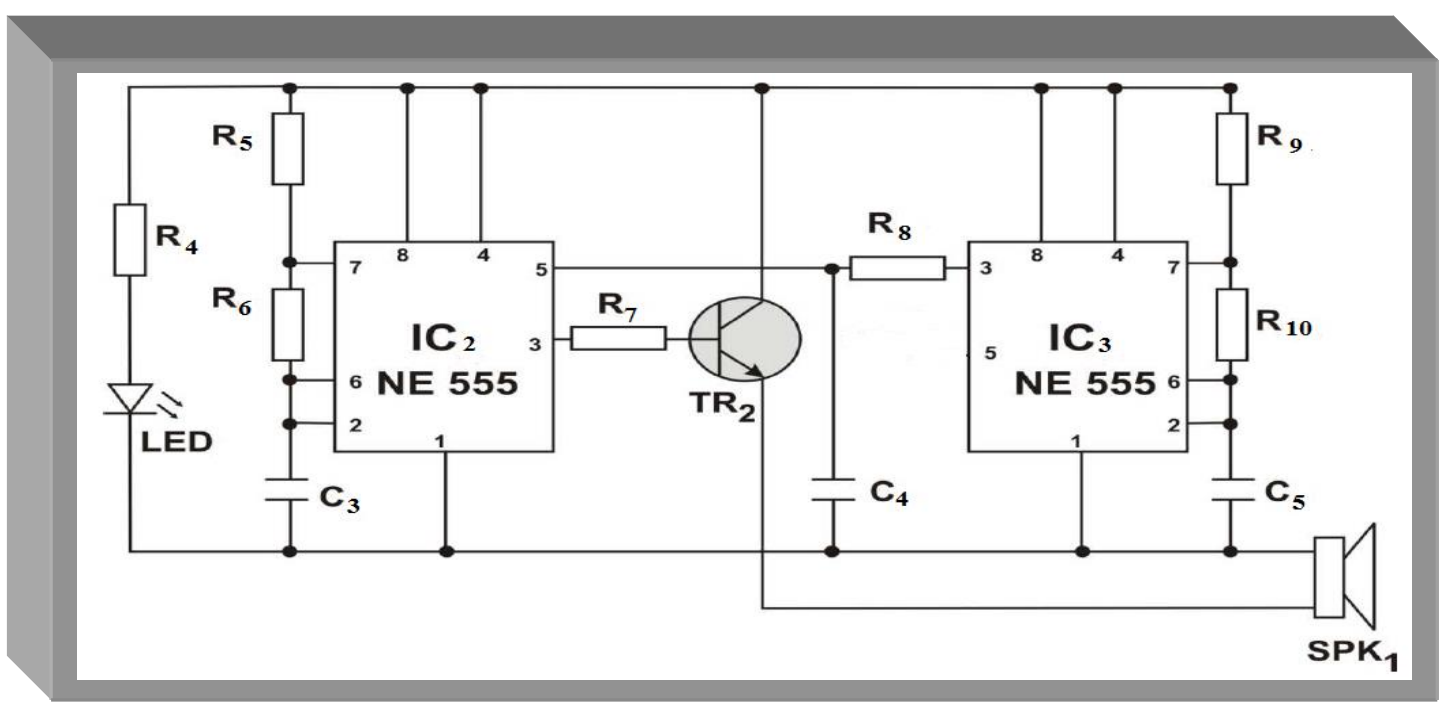

Figure 5. Circuit Diagram of the Alarm Unit

The figure above shows the alarm unit, having 4 basic components or sub-units which are; Two 555 timers IC2 and IC3 operating in a stable mode to produce a siren sound, a power transistor (TR2) used for further amplification of the audio output and a LoudSpeaker $\left(\mathrm{SPK}_{1}\right)$ that transduces the output signal to audible sound. IC2 operates at a high frequency of around $481 \mathrm{~Hz}$, and act as a voltage controlled oscillator, while producing a square wave. This forms the basic tone of the siren sound system. IC3 produces another square wave at much lower frequency of around $0.5 \mathrm{~Hz}$ which alters the rhythm of the steady tone from IC2 to the desired siren sound. The output of IC3 is actually coupled through R8 to control the voltage terminal of IC2. The low frequency output from IC3 is used to modulate the high frequency produced by IC 2 thereby alternating the frequency of operation of IC2 to produce a siren sound instead of a mono tone.

The final siren note is available at output pin 3 of IC2 which serves as input signal to the transistor TR2 for further amplification in order for the signal to be able to drive an $8 \Omega$ loud speaker and produce a very loud audible alarm sound.

\subsection{Power Supply}

The power unit is responsible for turning on the entire circuit. Some components are used to set up power unit and they include; a 12v step down transformer, a bridge rectifier circuit, a smoothening capacitor and a voltage regulator IC.

\subsubsection{Step-Down Transformer}

A transformer is a device consisting of two closely coupled coils called primary and secondary coils. An AC voltages applied to the primary appears across the secondary with a voltage multiplication proportional to the primary which appears across the secondary with a voltage multiplication proportion to the turn ratio of the transformer and a current multiplication inversely proportional to the turn ratio. Power is conserved.

$$
\text { Turn Ratio }=\frac{\mathrm{VP}}{\mathrm{VS}}=\frac{\mathrm{NP}}{\mathrm{NS}}
$$

and 
Where, $\mathrm{VP}=$ primary voltage

$\mathrm{Vs}=$ Secondary output voltage

$\mathrm{NP}=$ number of turns in primary coil

$\mathrm{NS}=$ number of turns on secondary coil

$\mathrm{IP}=$ primary input current

Is $=$ Secondary output current

For the 12 volts step down transformer needed for this project, the turn ratio is 240:12 it is represented as shown in figure 6 below.

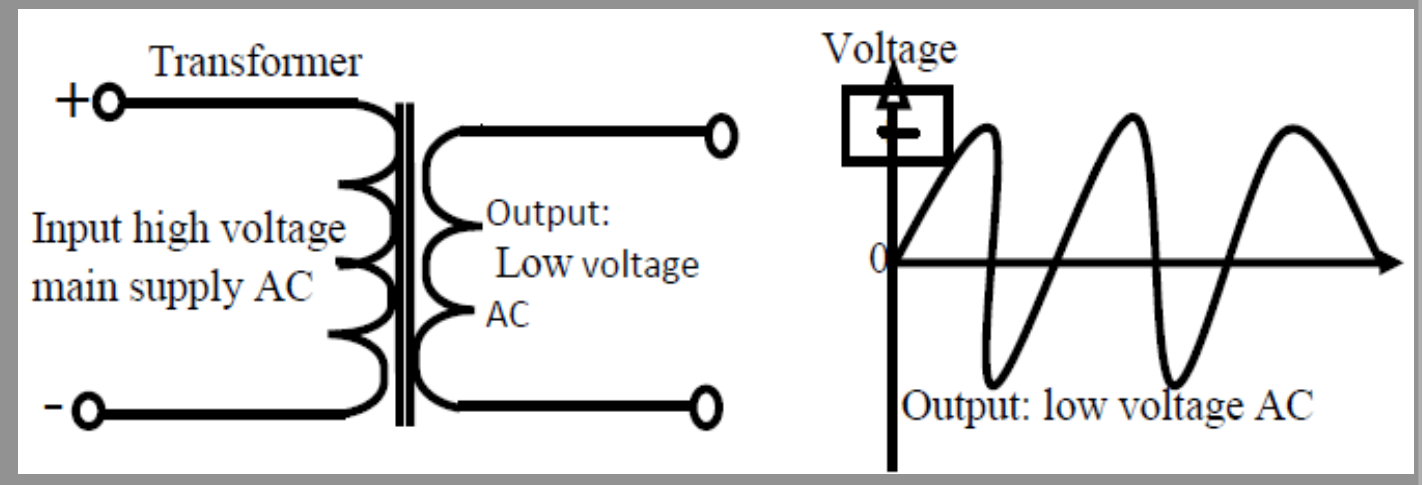

Figure 6. Transformer Circuit Representation

\subsubsection{Rectifier Stage}

Here, a rectifier is necessary to converts the $12 \mathrm{~V}$ (A.C) supply from the transformer into a pulsating D.C voltage. A full bridge rectifier was used for this purpose. It consist of four diodes (IN 4001 series) arranged as shown in figure 7 below. During the positive half cycles diodes D2 and D3 are forward biased and current flows through the terminals. In the negative half cycle, diodes D1 and D4 are forward biased. Since load current is in the same direction in both half cycles, full wave rectifier signal appears across the terminals [6].

So, a bridge rectifier circuit (figure 7) was constructed using four individual diodes, though; it is also available in special packages containing the four diodes required. It is called full-wave rectifier because it uses the entire AC waves (both positive and negative section) $1.4 \mathrm{v}$ is used up in the bridge rectifier because each diode uses $0.7 \mathrm{v}$ when diodes conducting as shown in the figure below. The maximum current they can pass rates bridge rectifiers and the maximum reverse voltage they can withstand this must be at least be three times the supply RMS voltages so, the rectifier can withstands the peak voltage. 


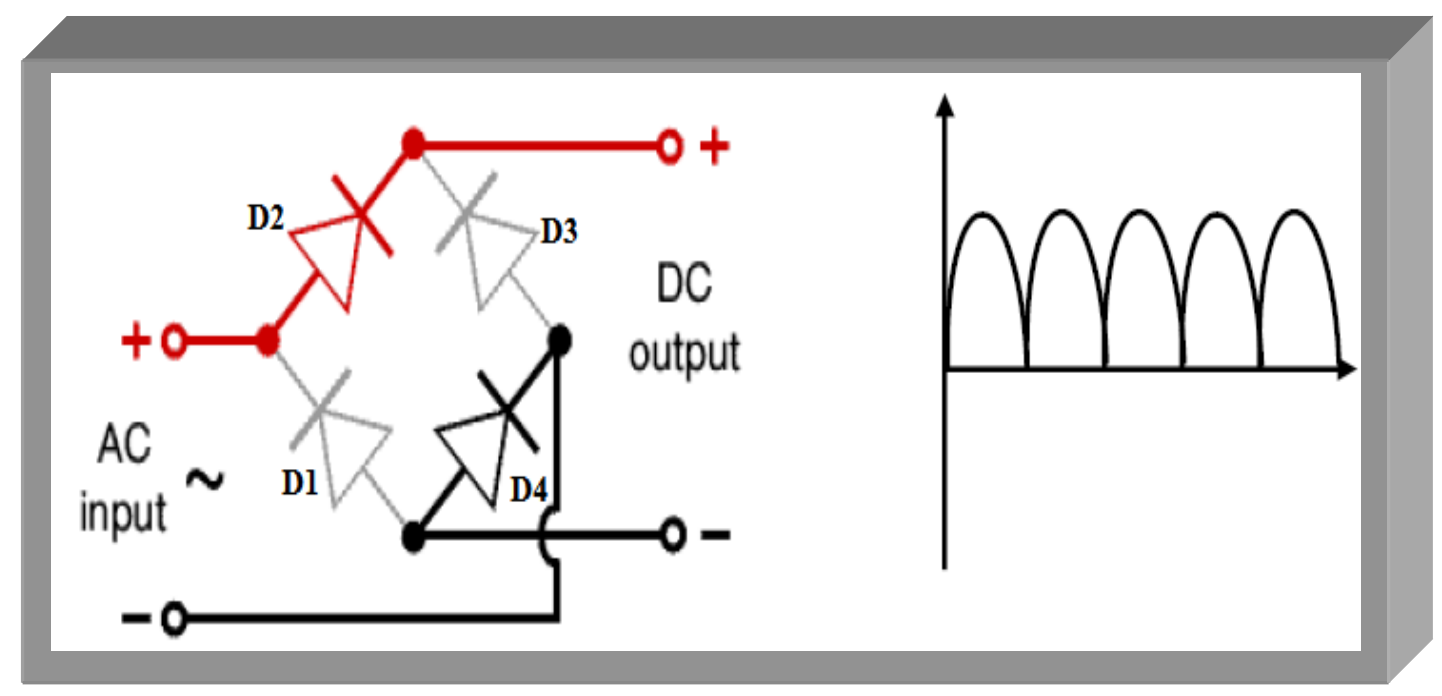

Figure 7. Bridge Rectifier

Alternate pairs of diode conduct changing over the connections so the alternating directions of $\mathrm{AC}$ are converted to the direction of DC.

\subsubsection{Filter Stage}

The pulsating D.C voltage that comes out from the rectifier stage is converted into constant D.C voltage with the aid of a filter capacitor (figure 8). This capacitor is large value electrolytic capacitor. It charges up - that is, stores energy - during the conduction half cycle thereby opposing any changes in voltage. The filter stage therefore filters out voltage pulsations (or ripple).

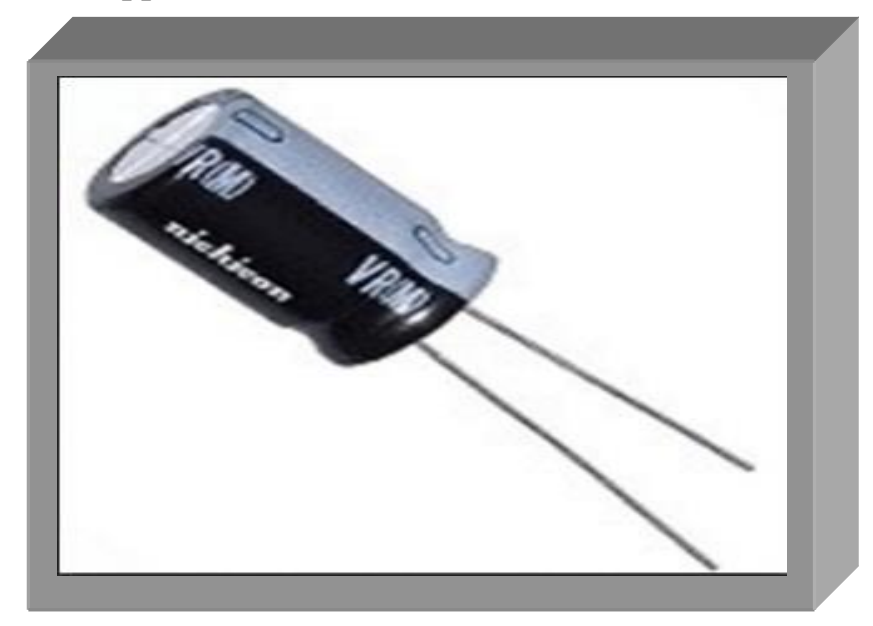

Figure 8. Filter Capacitor

\subsubsection{Voltage Regulator}

A voltage regulator also called a "regulator" has only three legs and appears to be a comparatively simple device but it is actually a very complex integrated circuit. A regulator converts varying input voltages and produces a constant "regulated" output voltage. Voltage regulators are available in a variety of outputs.

The output of the filter stage varies slightly when the load current or output voltage varies and it is a $12 \mathrm{~V}$ D.C supply which is higher than the circuit requirement. For these 
reasons, a Voltage Regulator was required to stabilize the voltage and also reduce it from $12 \mathrm{~V}$ to a $5 \mathrm{~V}$ steady D.C supply.

And, for this project, the basic L7805 voltage regulator (figure 9) was selected - a three-terminal positive regulator with a $5 \mathrm{~V}$ fixed output voltage - for its ability to eliminate distribution problems associated with single point regulation. This fixed regulator provides a local regulation, internal current limiting, thermal shut-down control, and safe area protection making it essentially perfect and indestructible for our project. With adequate heat sinking, an L7805 voltage regulator is capable of delivering over 1A output current.

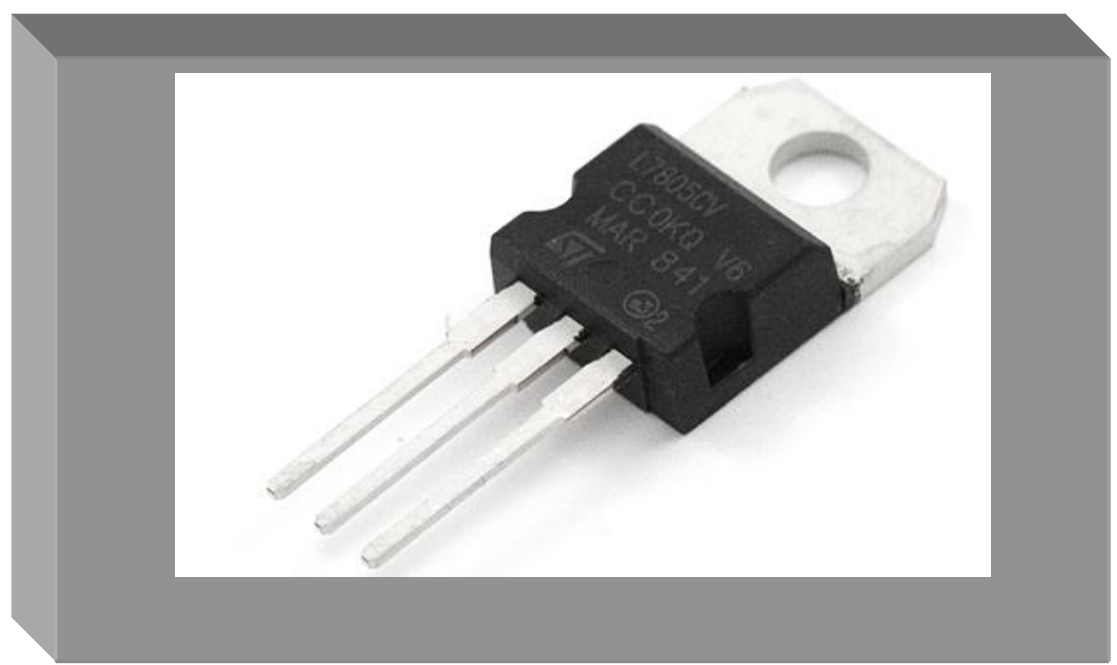

Figure 9. L7805

\section{Results and Discussion}

The Intelligent Intruder Alert System was constructed according to its design and was tested before and after housing accordingly. That is, the sensor was setup at the back of a door and the other part installed appropriately, then, the whole system was powered, after few seconds, the door under surveillance was opened, at this instance an audible soundAlert- sounded and was heard which signified the opening of the said door by an intruder.

From these steps, it was evident that this system can sense/detect the opening of a door under surveillance or intrusion, wherever it is installed and sound Alert to inform/warn residence of the presence of an intruder. Therefore, the result was as expected.

The test result shows that after powering/switching on the Intelligent Alert System and the door closed, nothing happened, but as soon as somebody opened the monitored door, the system triggered, an alert sounded and the sound remained on irrespective of whether the door is quickly closed or left opened except on the condition that the sound is deactivated by the reset switch. This shows that the device is working perfectly.

\section{Conclusions}

It can be concluded that our overall aim of this project which was to design and construct an Intelligent Intruder Alert System was achieved, in that our specific objective, that was to develop a cheap, affordable, reliable and efficient security system, was successfully realized at the end of the design, construction and implementation processes. One factor that accounts for the cheapness of the product was the proper choice of components used. The ones that were readily available were used, while close substitutes were found for those that were not readily available. The system was tested and found to be working to specifications and predictions. Summarily, a cheap and reliable way of checking the activities of burglars and intruders has been successfully developed, which is 
the aim of this research. It can conclusively be said therefore, that the benefits of having this Intelligent Intruder Alert System cannot be overemphasized.

We therefore recommend that; this work can be improved upon to cover wider or longer range and outlets, sends Short Message Services (SMS) alert to cell phone as a theft alarm, call property owner's mobile phone number or police line, Security camera could also be incorporated to record intruder's footage and activities. It could otherwise be modified to monitor patient's movement or elderly in hospital or at home.

\section{Acknowledgements}

Thanks to IGBAMERUN Sogo for sourcing some of the components used.

\section{References}

[1] "Burglary a long standing social menace". Available at: www.eaglesecurity solutions.co.uk/blogs/burglary-a-long-standing-social-menace/ Accessed; Jan. 8, 2017.

[2] "Why You Should Consider Door Entry Systems". Available at: www.eaglesecurity solutions.co.uk/blogs/why-you-should-consider-door-entry-systems/ Accessed; Jan. 8, 2017.

[3] How Burglar Alarms work, Available at: http://www.howstuffworks.com, 2016.

[4] K. Akinloye Oyelakin, Design and Construction of a World-Wide-Mobile-Call-Back Burglar Alarm System, International Journal of Innovation and Scientific Research, Vol. 12 No. 1 Nov. 2014, pp. 6569.

[5] Nick A. Tim, "Technical leaflet on introduction to fire detection and Alarm", Available at: http://www.neddc.org/alarm.html.

[6] J. Shepherd, A.H Morton and L.F Spence, "Basic Electrical Engineering”, Pitman Publishing Reprinted 1973, Page 274-278.

\section{Authors}

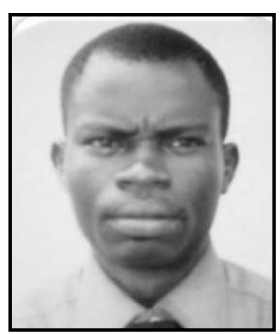

Oyelakin, K. Akinloye, received his Master of Technology (M.Tech) from The Federal University of Technology (FUT) Akure, Nigeria in 2014 and a Postgraduate Diploma in Physics and Electronics, from the same University, in 2006 having obtained his undergraduate - in 2002 - in the same field. A member of academics of Rufus Giwa Polytechnic, Owo, Nigeria, whose fields of interest span Instrumentation, Electronic Projects, Space/Environmental Research, Security, swarm intelligence and Physics as a whole, he has published research papers in Refereed International Journals. And, he belongs to the Space Research Group of the Federal University of Technology, Akure, Ondo State, Nigeria.

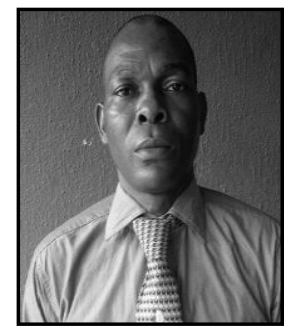

Egbuwalo, Shadrack A., received his Postgraduate Diploma in Physics/Electronics (PgD, 2015), Postgraduate Diploma in Computer Science (PgD, 2008) both from the Federal University of Technology, Akure, Nigeria and an Higher National Diploma in Physics with Engineering Production (HND, 2002), from the Nigerian Institute of Science Laboratory Technology, Ibadan, Nigeria. He is currently a member of academic staff of Rufus Giwa Polytechnic, Owo, Ondo State, Nigeria. 
International Journal of Security and Its Application Vol.11, No.2 (2017) 